\title{
A study of cellular structures under impact loading
}

\author{
S. Abdennadher, H. Zhao and R. Othman ${ }^{1}$ \\ LMT, ENS-Cachan/CNRS, Université Paris 6, 61 avenue du Président Wilson, \\ 94235 Cachan cedex, France \\ ${ }^{1}$ LMS, École Polytechnique, 91120 Palaiseau cedex, France
}

\begin{abstract}
This paper presents the effect of inertia of cellular structures under impact loading. Experimental results under static and dynamic loading will be presented for square tubes made of a rate quasi-insensitive material (brass), the dynamic results being obtained using modified split Hopkinson pressure bar technique. The numeric simulation under Ls-dyna of those tests giving similar results will be also presented. Presented experimental, numeric results show that the inertia effect is a dominant factor responsible for the enhancement of crushing strength of cellular structures.
\end{abstract}

\section{INTRODUCTION}

It is generally observed that many cellular materials show a significant enhancement of their strength under dynamic crushing loading. This strength augmentation is often supposed due to the behaviour enhancement at high strain rates of base-materials, of which cellular structures are made. However, some recent experimental works show that the proportion of such an enhancement due to basic material behaviour is largely inferior to that observed for corresponding cellular structures $[1,2]$.

In this paper, the effect of inertia of cellular structures under impact loading will be presented. The progressive folding being the major crushing mechanism of cellular structures, the inertia effect in the folding process is then studied. For this purpose, square tubes are used here as a model structure, the crushing of which is a progressive one $[3,4,5]$. In section 2 , experimental results under static and dynamic loading will be presented for the base materials (brass) and for square tubes. The increase of crushing strength is observed for brass tubes whereas basic materials are rate insensitive. It proves that the constitutive material behaviour is not the only source of strength enhancement. In section 3, numeric simulation under Ls-dyna of those tests giving similar results will be also presented. The implicit version of the code is used for quasi-static test. The same mesh size and same type of plate element is used in both static and dynamic simulation. The local information obtained by these numeric tests show how the inertia effect works in the folding process. In section 4 , an experimental validation by post mortem Vickers micro hardness measurement for various stages of tube crushing will be given.

\section{STATIC AND DYNAMIC CRUSHING TEST OF BRASS SQUARE TUBE}

The commercial brass square tube $(35 \times 35 \mathrm{~mm})$ of $1.5 \mathrm{~mm}$ thick have been chosen for this study because the brass is supposed to be rate insensitive and the density is high. However, an important strain hardening during producing process makes those tubes quite brittle and the progressive crushing mode is not observed because of the early fracture at the four edges. 
Annealing becomes then indispensable to make them more ductile. In order to obtain a stable material property after the heat treatment, the exact chemical composition has been determined $(\mathrm{Cu} 64 \%, \mathrm{Zn} \mathrm{36 \% )}$ by the EDS analysis. According to the phase equilibrium diagram, the suitable heat treatment is following: 30 min annealing at $450^{\circ} \mathrm{C}$ and the water quenching to keep the microstructure.

This heat treatment guaranties a good reproducibility of material properties. Indeed, static crushing tests have been performed on treated tubes with a MTS-testing machine. In order to avoid the global elastic buckling mode, the length of the tube specimen is $104 \mathrm{~mm}$. The progressive folding mode is observed this time and force-displacement recordings are quite repeatable (Fig1).

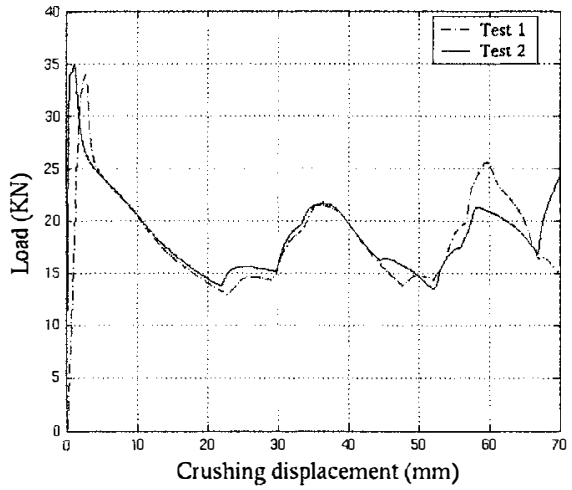

Figure 1. Reproducible static crashing tests of brass tubes

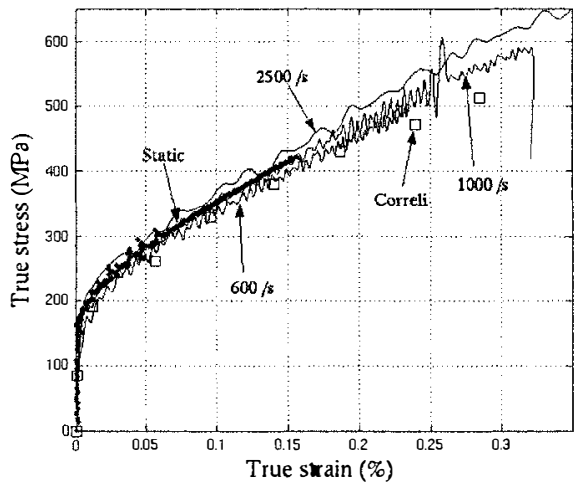

Figure 2. Stress strain relations at static and dynamic strain rates

In order to know if the basic material is really rate insensitive, especially after annealing, static and dynamic compression tests are performed, using very small specimen to avoid the buckling of the specimen. We prefer compression test with SHPB than tension test for the reason of the measuring accuracy and attaching difficulties under impact loading. The specimen is cut from the tube, the dimension of which is of $2 \mathrm{~mm}$ high and about $10 \mathrm{~mm}$ width. Dynamic test with SHPB can be made without many experimental difficulties because necessary attention on the data processing is paid [6].

For the static compression test, the measurement of the strain is very difficult as the displacement is small and there is no place to put a strain gage. An optical measurement is made to control the quality of displacement recording. This technique is based on the comparison of image taken during the test with a remote microscope. Suitable image processing (Correli) can provide a precision of calculated strain at least $10^{-4}[7,8]$. Figure 2 shows a comparison of strain stress relations at the quasi static rate and high strain rates up to $2500 / \mathrm{s}$. It is clear that there is no rate sensitivity.

For the dynamic crushing test, an original testing configuration is developed to perform dynamic crushing test. Indeed, most of testing results reported in the literature are obtained with falling weight device, because of the energy needed in the crushing and size of the tube. The deceleration of that mass is measured by an accelerometer. The associated force and displacement are then deduced, but the measuring precision is not always very good because of the vibrations of the weight and its suspension system. Thus, previous works in open literature report often just the final crushing displacement at a given impacting mass and velocity. An 
accurate force measurement during the test is really desired to compare with static test. It is thus of interest to use a SHPB system, known for its measuring precision, to test these kinds of structures. However, this application requires a SHPB bar with a large diameter to cover the tube section. A large diameter $(80 \mathrm{~mm})$ SHPB system is used for this application. In order to get a maximum direct measurement, he direct impact Hopkinson bar test configuration is used, putting the input bar in contact with output bar. It gives a measurement of about $4 \mathrm{~ms}$ (a round-trip of $20 \mathrm{~m})$.

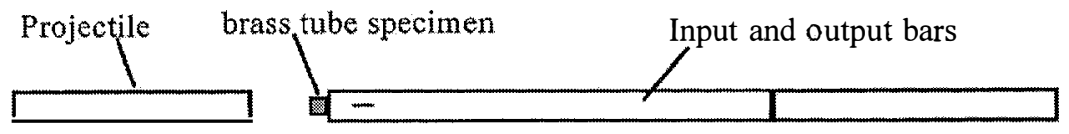

Figure 3. Direct impact SHPB test configuration

The velocity at the impacting face is then calculated by the following equation.

$$
V_{\text {input }}(t)=V_{0}-\frac{S_{p} E}{S_{p} \rho_{p} C_{p}}\left(\varepsilon_{t}(t)+\sum_{n=1}^{N} 2 \varepsilon_{t}(t-n T)\right)
$$

$$
\text { if } \mathrm{NT} \leq \mathrm{t}<(\mathrm{N}+1) \mathrm{T}
$$

where $S_{p}, \rho_{p}, C_{p}$ are respectively the cross-sectional area, the density and the wave velocity of the projectile. $T=2 l_{p} / C_{p}$, being the characteristic time (time needed for the wave to perform a round trip in the projectile).

Figure 4 shows testing results on brass square tubes with the same heat treatment (35x35mm, thickness of $1.5 \mathrm{~mm})$. The comparison between static and dynamic testing results illustrates a significant enhancement of crushing forces.

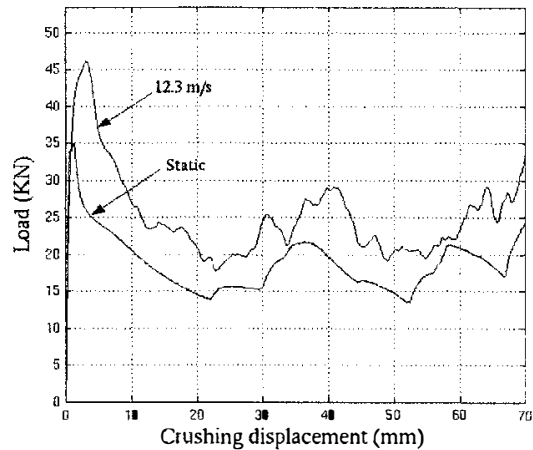

Figure 4. Force displacement curves under static and dynamic loading

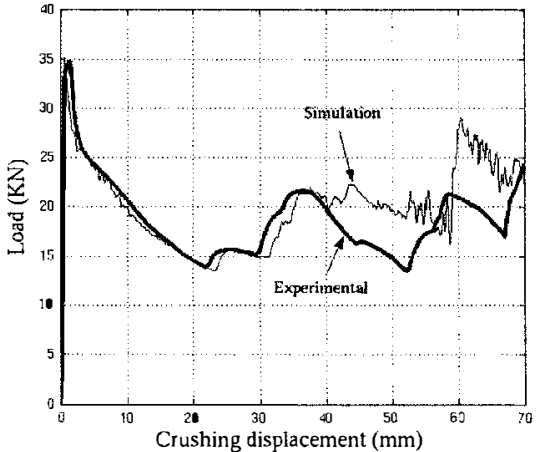

Figure 5. Comparison between simulated and experimental curves

\section{NUMERICAL SIMULATIONS UNDER STATIC AND DYNAMIC LOADING}

From the experimental evidence above, a numerical study based on the simulation of those tests is presented, using a rate insensitive constitutive law identified from tests presented in above 
section on the small compression specimen. Numerical results presented here are obtained using a commercial code (Ls-Dyna). The constitutive law is modelled as a Johnson-cook type law available in the code. Parameters for the rate sensitivity and the temperature effect in Johnson cook law are chosen as zero.

The static simulation is performed with the implicit version of Ls-Dyna. For the dynamic test, the measured velocity at both sides of the specimen in the SHPB test is imposed for the calculation with the explicit version. The used mesh and plate elements are the same. The figure 5 shows a quite good correspondence between experimental and numerical force-displacement curves under static loading. Compared with experimental pictures, the numerical calculation gives a fair reconstitution of the events (Fig. 6).

Such a numeric test allows for a careful examination of the crushing mode, event sequence, and especially local stresses and strains which can not be measured during the test.
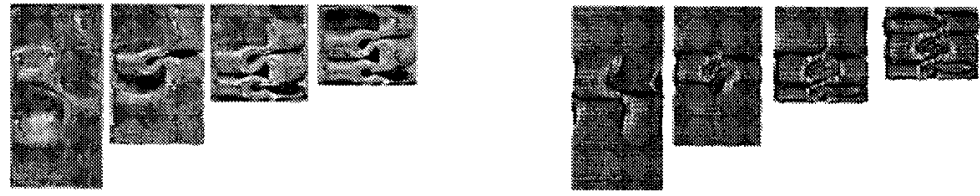

Figure 6. Comparison between simulation and tests

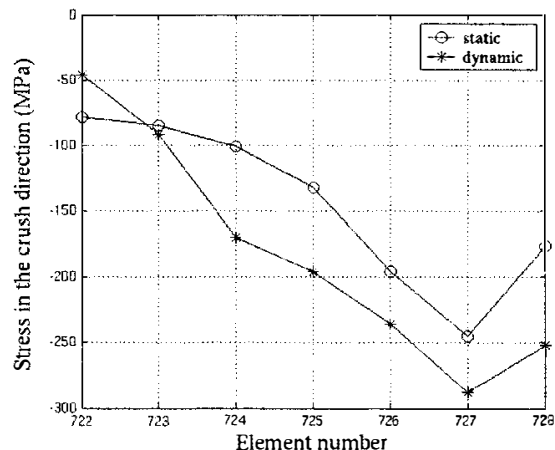

Figure 7a. Stress profile from centre to corner

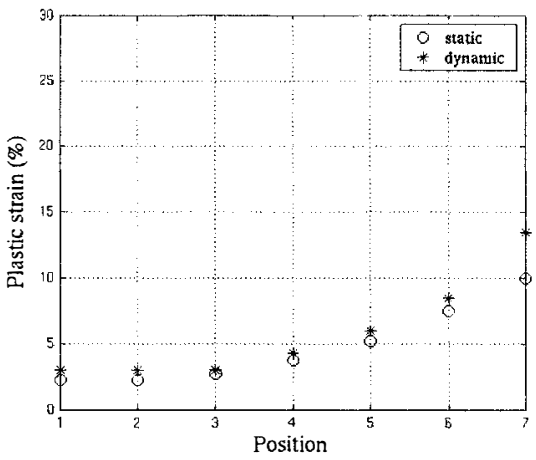

Figure 7b. Residual strain profile

A careful examination of the stress in the cross sections of tube and crushing profiles reveals that there exist small areas near the corners remains nearly straight while the middle of plate is seriously deformed. The corner elements support then most of the crushing loading and the buckling of such small areas correspond to the falling of the crushing loading. It means that the buckling force of these zones determine the peak loading in the folding process. In order to illustrate this, figure 7a gives the profile of the stress (in the direction of crushing) in the cross section cutting through these small zones just before their buckling. We can see easily that in the dynamic case, the compressive stress is higher than the static one. It reveals that in these small areas, elements are more compressed under dynamic loading. This point is clearly shown in the figure $7 \mathrm{~b}$ which illustrates the middle surface residual strain profile in this section. The buckling 
theory for a straight beam under dynamic loading applies then here [10, 11], which explain finally why such a strength enhancement under impact loading.

\section{VALIDATION BY MICRO HARDNESS MEASUREMENT}

Above local information given by the simulation shows clearly where the origin of this strength enhancement under the dynamic loading is. However, this theory based on the local simulated information should be experimentally proved. For this purpose, the micro hardness test on crushed tubes at various crushing stages have been done. It provides then experimental examination of local informations given by simulations. Indeed, the micro hardness of the material measured from a micro-indentation test reveals an average strain hardening state of the material. Even the relation between hardness and hardening state is quantitatively related, it will give at least a qualitative comparison of hardening state for the same points between static and dynamic tests. It could give also the relative evolution in different points for a given test. Furthermore, if reference tests on the basic material at given strain hardening are performed, a quantitative relationship can be derived for this material.

Actually we do know the nominal strain for the small compressive tests performed in order to establish a constitutive law, post mortem indentation tests on these small specimens gives a correspondence between the size of stump of indentor and the known nominal plastic strain. This correspondence for treated brass material is shown in figure 8a. The same micro hardness measurement (same machine) will be applied to the square tubes afterwards.

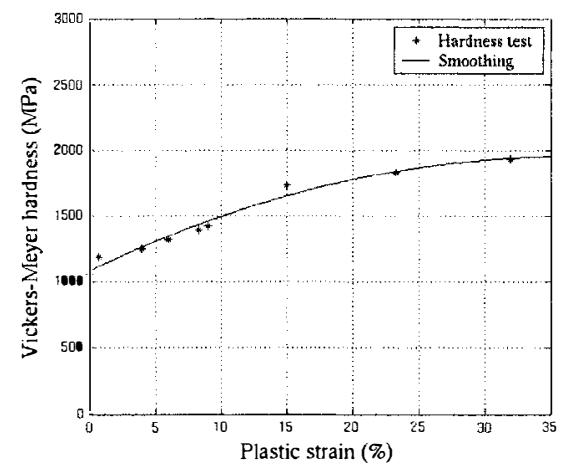

Figure 8a. Correspondance hardness vs. plastic strain

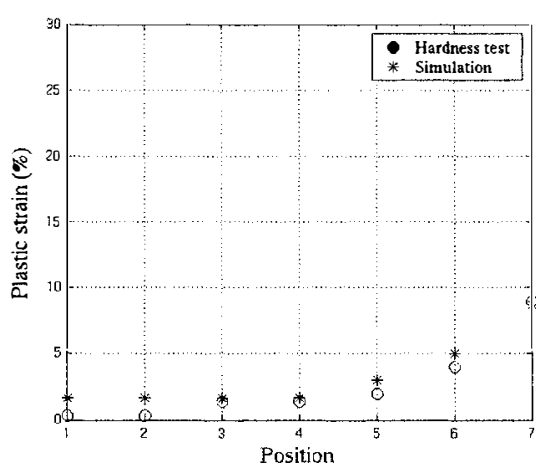

Figure 8b. Hardness measurement compared with simulation

Qualitative and quantitative matching can be then made between simulated information and experiments. Three crushing states under static loading are used for this matching. The first one just after the first peak load, the second just after the second peak load which will be the progressive peak load. The last one is after full crashing and we compared also the specimen fully crushed under dynamic loading. Figure 8b shows a typical simulation measurement comparison.

The excellent match between simulation and post mortem microhardness measurement confirms then features given by simulations. After the first peak load, the main crushing load is supported in a small area near the corner, the buckling of which leads to the force drop down and determine consequently the corresponding progressive peak load. 


\section{CONCLUSION AND PERSPECTIVE}

In summary, accurate experimental results show a significant enhancement of crushing strength for square tubes made a $\mathrm{Cu}-\mathrm{Zn}$ alloy which are proved to be rate insensitive. Numerical simulations using a rate insensitive constitutive law confirm this enhancement. Careful examinations of the stress and strain profiles show that existing small zone near the corner of tubes remain straight and support the main loading in the folding process. The theory of dynamic plastic buckling of straight beam can be applied. It leads then to the conclusion that the inertia effect is a dominant factor responsible for the enhancement of crushing strength of studied tubes. Post mortem microhardness measurement in various crushing stages confirms this conclusion.

Such an idea can be easily applied to the cellular structures such as the honeycomb. Figure 9 shows a comparison between test and simulation for a representative cell of an aluminium honeycomb.

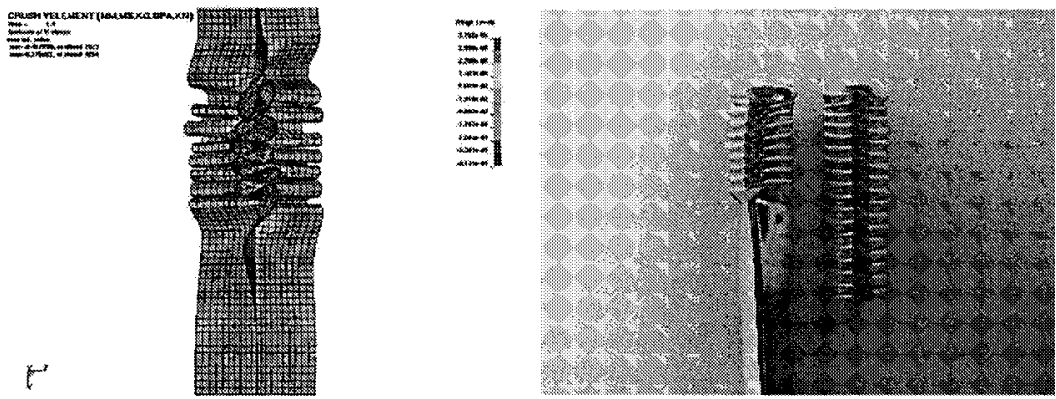

Figure 9. Comparison between experiments and simulations for honeycomb.

\section{References}

[1] H. Zhao and G. Gary, Int. J. Impact Engng, 21 (1998), 827-836.

[2] E.Wu, W.S. Jiang, Int. J. Impact engng. Vol.19, pp439-456, 1997

[3] W. Abramowicz and N. Jones, Int. J. Impact Engng, vol 2, pp. 179-208, 1984.

[4] W. Abramowicz and T. Wierzbicki, J. of applied mechanics, 56, 3, (1989), pp. 113-120.

[5] M. Langseth and O.S. Hopperstad, Int. J. Impact Engng, 18, 7-8, pp. 949-968, 1996.

[6] H. Zhao and G. Gary, Int. J. Solids \& Structures, vol.33, pp3363-3375, 1996.

[7] F. Hild, J.-N. Périé et M. Coret, CORRELI. LMT-Cachan, rapport interne n 230, 1999

[8] L. Chevalier, S. Calloch, F. Hild et Y. Marco, Eur. J. Mech. A/Solids, vol.20, pp169-187, 2001

[9] H. Zhao and G. Gary, J. Mech. Phys. Solids. Vol.45, pp1 185-1202, 1997.

[11] C.R. Calladine and R.W. English, Int. J. Mech. Sci., vol. 26, pp. 689-701,1984.

[12] G. Gary, Modélisation et étude expérimentale du flambement dynamique, Thèse d'état, 1980. 